\title{
Formation of Pancreatoduodenal Fistula in Intraductal Papillary Mucinous Neoplasm of the Pancreas Decreased the Frequency of Recurrent Pancreatitis
}

\author{
Gebran Khneizera, d, Kavya M. Reddy ${ }^{\mathrm{b}}$, Muhammad B. Hammamic, \\ Samer Alkaade ${ }^{\mathrm{b}}$
}

\begin{abstract}
Intraductal papillary mucinous neoplasms (IPMN) of the pancreas are characterized by proliferation of mucin-secreting cells in the main pancreatic duct (PD) or its branches. The secreted thick mucin usually leads to PD obstruction and dilation. A common complication of IPMN is recurrent acute pancreatitis secondary to poor pancreatic fluid drainage, and rarely, pancreatobiliary and pancreatointestinal fistulae. We describe a unique case of IPMN in a 57-year-old male who was referred to our institution for evaluation of recurrent acute pancreatitis. After extensive evaluation, he was diagnosed with main duct IPMN. Intraductal PD biopsy revealed intestinal type IPMN with intermediate grade dysplasia. Patient was managed clinically by large caliber (10 French) PD stenting which eliminated his recurrent acute pancreatitis. The patient was initially referred for pancreatic resection; however, surgery was aborted and evaluated to be high risk with high morbidity secondary to the extensive adhesions between the pancreas and surrounding structures. Patient remained clinically stable for a few years except for an episode of acute pancreatitis that happened after a trial of stent removal. Subsequently, the patient did well after the PD stent was replaced. Recently, repeat abdominal imaging revealed a large pancreatoduodenal fistula which was confirmed on repeat endoscopic retrograde cholangiopancreatography. We were able to perform pancreatoscopy by advancing a regular upper scope through the fistula and into the PD. Interestingly, the fistula relieved the symptoms of obstruction and subsequently decreased the frequency of recurrent pancreatitis episodes with no further episodes at 6 months follow-up. This case highlights the importance of providing adequate PD drainage to reduce the frequency of recurrent acute pancreatitis in the setting of main duct IPMN, especially if the patient is not a surgical candidate. Also, physicians need to monitor for compli-
\end{abstract}

Manuscript submitted January 6, 2019, accepted February 1, 2019

andiana University School of Medicine, Indianapolis, IN, USA

bSaint Louis University School of Medicine, St. Louis, MO, USA

'University of California San Francisco School of Medicine, San Francisco, CA, USA

${ }^{\mathrm{d} C}$ Corresponding Author: Gebran Khneizer, Department of Internal Medicine, Indiana University School of Medicine, IU Health University Hospital and Physicians, 550 N University Blvd, Suite 1501, Indianapolis, IN 46202, USA. Email: gkhneizer@iuhealth.org

doi: https://doi.org/10.14740/gr1140 cations such as fistula formation between the pancreas and surrounding structures in the setting of chronic inflammation due to recurrent episodes of pancreatitis. Early identification of a fistula is important for surgical planning. Furthermore, since recent studies suggested a higher incidence of additional primary malignancies in patients with IPMN of the pancreas compared to the general population, patients may be considered for screening for other primary malignancies.

Keywords: Intraductal papillary mucinous neoplasms of the pancreas; IPMN; Fistula; Recurrent pancreatitis

\section{Introduction}

Intraductal papillary mucinous neoplasms (IPMN) of the pancreas are tumors that originate in the main pancreatic duct (PD), branch ducts, or both. They are characterized by intraductal proliferation of mucin-secreting cells that leads to pancreatic ductal strictures/obstruction with resultant upstream dilation of the involved PD or branch. Detection of these lesions has increased over the past decade due to the widespread use of advanced abdominal imaging modalities which are performed for evaluation of pancreatic or non-pancreatic symptoms. A common complication of IPMN is recurrent episodes of acute pancreatitis due to pancreatic ductal obstruction/stricture with impairment of pancreatic fluid drainage [1]. Furthermore, there have been rare reports of pancreatobiliary and pancreatointestinal fistulae associated with IPMN in the literature, and when reported, these cases are usually seen in the elderly population.

We present a unique case of IPMN complicated by large fistula formation between the pancreas and the small bowel in a middle-aged man with episodes of recurrent pancreatitis, in which the fistula relieved the symptoms of pancreatic ductal obstruction and subsequently decreased the frequency of the recurrent attacks of acute pancreatitis.

\section{Case Report}

A 57-year-old male with history of hypertension, gastroesophageal reflux disease, prostate cancer status post total prostatectomy, and type 2 diabetes mellitus presented for evaluation of 


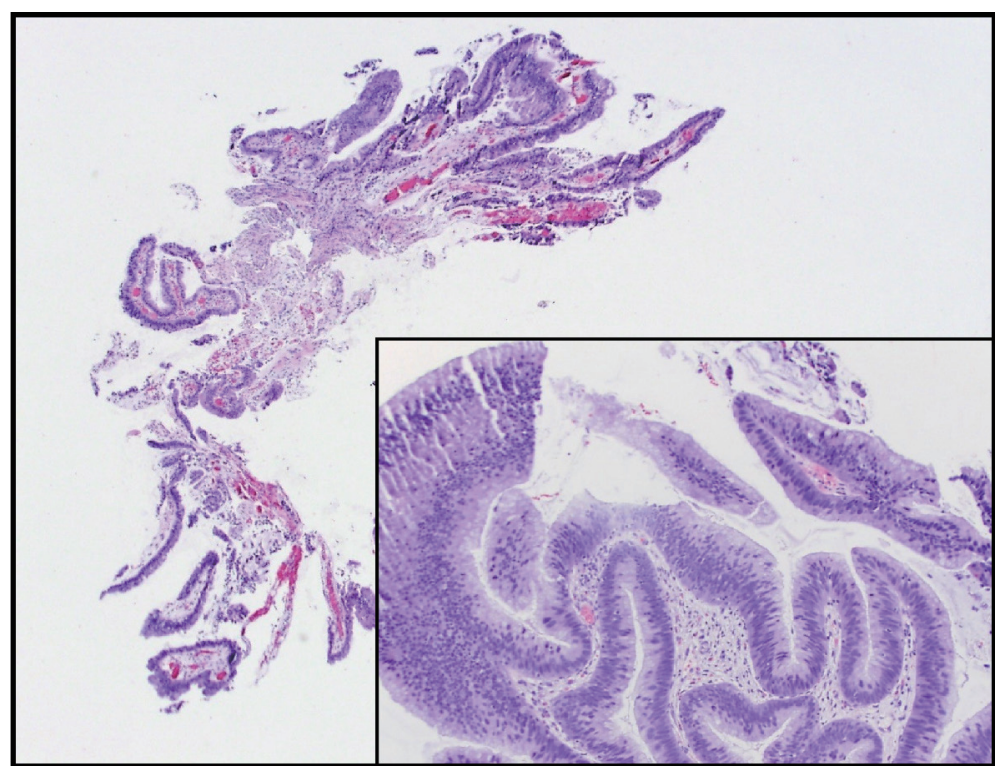

Figure 1. This is a section of the pancreatic duct biopsy showing mucinous epithelium with a papillary architecture. The higher power view (inset) shows areas with nuclear hyperchromasia and crowding, consistent with intermediate grade dysplasia.

chronic pancreatitis and unintentional weight loss of 40 pounds over a 6-month period. The patient's social history was significant for alcohol use (three drinks per week) that he quit 5 years prior to presentation and chewing tobacco. Patient's medications included pancrelipase 24,000 units twice daily, insulin detemir, omeprazole and losartan. His vital signs on presentation were remarkable for a blood pressure of $173 / 81 \mathrm{~mm} \mathrm{Hg}$. Physical examination was unremarkable except for a healed scar at the right upper quadrant. Three years prior to this presentation, the patient was diagnosed with acute pancreatitis which was thought to be secondary to gallstones and his initial course was complicated by a ruptured gallbladder which required open cholecystectomy. He subsequently developed five episodes of pancreatitis over the span of 6 months before presentation, with the frequency of recurrence increasing to every 3 - 4 weeks.

The patient underwent a magnetic resonance cholangiopancreatography (MRCP), which showed marked dilatation of the main PD with mild ductal irregularity and diffuse side branch dilatation, and the main PD was narrowed at the site of insertion into the duodenal papilla. There was also a mention of groove pancreatitis (pancreatitis affecting the groove between the pancreatic head, duodenum, and common bile duct) by visualization of wall thickening and cystic changes of the second portion of duodenum with surrounding inflammatory fat stranding. Therefore, endoscopic ultrasound (EUS) and endoscopic retrograde cholangiopancreatography (ERCP) were performed and revealed changes consistent with severe chronic pancreatitis classified as Cambridge grade 5, along with main PD dilation to $13 \mathrm{~mm}$ in diameter as well as irregularities and stricture in the mid body of pancreas. In addition, hyperechoic strands and intraductal filling defects suggestive of stones were visualized and the common bile duct (CBD) was dilated up to $10 \mathrm{~mm}$ with mild narrowing in the head of the pancreas. Pancreatic and biliary sphincterotomies were performed and thick mucin, debris, and small stones were removed from the main PD. Brushing and biopsies were obtained from the PD stricture followed by placement of a plastic stent (10 French) in the main PD and another plastic ZSO 10-9 stent in the CBD. Histopathology examination revealed pancreatobiliary mucosa with chronic active inflammation and submucosal fibrosis and cytology revealed atypical cells favoring reactive changes.

Two weeks after the procedure, the patient developed another flare of acute pancreatitis. He subsequently underwent five ERCP procedures over the span of 14 months with multiple exchanges of main PD and CBD stents and removal of stones, mucous, and debris. Further brushings and biopsies of the PD stricture were obtained. After the procedures, the patient reported subjective improvement of his symptoms and remained for the most part symptoms free in between the ERCP procedures as long as the pancreatic stents had remained in place. Patient had few episodes of acute pancreatitis that were related either to migration or occlusion of the pancreatic stents. Subsequent biopsies revealed pancreatobiliary mucosa and low grade pancreatic intraepithelial neoplasia with papillary protrusion consistent with IPMN. The pathology specimen obtained during the last ERCP showed intestinal type IPMN with low grade dysplasia as well as areas of nuclear crowding, pseudostratification, and focal loss of polarity consistent with intermediate grade dysplasia (Fig. 1). There was no high-grade dysplasia or carcinoma identified.

Patient was subsequently referred to the hepatobiliary surgical team to be evaluated for surgical intervention. Tumor markers were sent and showed normal carbohydrate antigen (CA) 19-9 and alpha-fetoprotein (AFP) and elevated carcinoembryonic antigen (CEA) $(5.8 \mathrm{ng} / \mathrm{mL})$. Repeat MRCP showed one of the PD side branches extended anteriorly and abutted the gastric body with significant gastric wall thickening and surrounding inflammatory changes. Patient's course was later complicated by hospitalization for another episode of acute pancreatitis with resultant formation of pancreatic ab- 


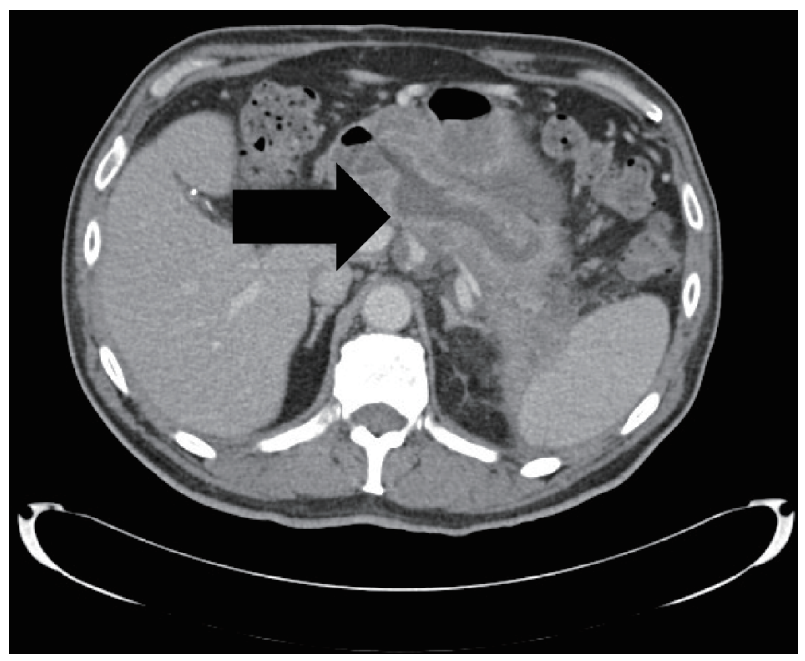

Figure 2. A sidebranch pancreatic duct within the pancreatic neck communicating with the first portion of the duodenum. It measures $1.6 \mathrm{~cm}$ in transverse diameter and represents a pancreaticoduodenal fistula.

scess which was communicating with the dilated main PD. He was treated with a 10-day course of ciprofloxacin and metronidazole. Patient was initially scheduled for distal or total pancreatectomy, but the surgery was delayed for 3 weeks to allow healing post hospitalization. Pre-operative computed tomography scan of the abdomen and pelvis with intravenous (IV) contrast demonstrated a pancreaticoduodenal fistula from the pancreatic neck to the first portion of the duodenum along with peri-pancreatic inflammatory changes and persistent ductal dilatation (Fig. 2). In the setting of the extensive adhesions between the pancreas, stomach and transverse mesocolon mesentery, the surgical procedure was aborted intraoperatively and will not be pursued further due to the high morbidity involved in attempting the pancreatic resection.

He later underwent another ERCP procedure which revealed a large pancreatoduodenal fistula at the bulb of the duodenum (Fig. 3). We were able to perform direct pancreatoscopy by advancing a therapeutic upper scope through the pancreatoduodenal fistula into the main PD. Diffuse villiform projections throughout the main PD consistent with main duct IPMN were visualized. The main PD was found to be diffusely dilated up to $22 \mathrm{~mm}$ and filled with thick mucus. Due to the good drainage of the PD through the fistula, we elected not to place a pancreatic stent. Patient remained clinically stable with no further episodes of recurrent acute pancreatitis for a few years.

\section{Discussion}

IPMN of the pancreas are mucinous tumors that originate in the main PD, branch duct or both [2]. Based on histological characteristics, they are divided into low, intermediate, high grade dysplasia and invasive carcinoma $[3,4]$. Frequency of incidental pancreatic cyst detection is on the rise secondary to the widespread use of imaging modalities which are done for other purposes [5]. Recent studies have described that IPMN are detected in $80 \%$ of patients with incidental pancreatic cysts

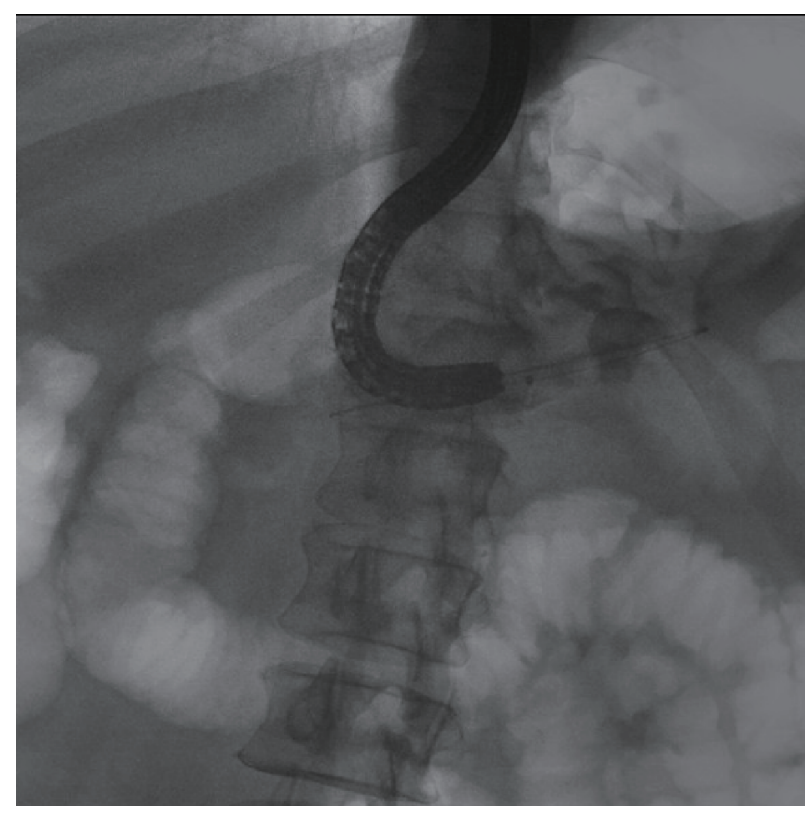

Figure 3. ERCP reveals the large pancreaticoduodenal fistula with contrast extravasation from the duodenum into the pancreatic duct.

[6]. The presentation of such cysts ranges from an asymptomatic incidentaloma [7] to a more severe clinical picture of acute pancreatitis manifesting as severe nausea, vomiting, and abdominal pain [8]. Once IPMN is suspected, specialized imaging modalities such as EUS and MRCP are performed to better visualize and characterize the cyst for malignant potential. Pancreatic cystic lesions with dilatation of the PD or one of its branches along with intraductal filling defects accompanied by chronic pancreatitis changes are the most frequently encountered findings on imaging [9]. Therefore, one of the main complications of IPMN is recurrent pancreatitis secondary to the obstruction of pancreatic fluid drainage.

Management of such neoplasms differs depending on the histologic classification and location. Literature has shown that branch duct IPMNs are less aggressive than their main duct counterparts for each given size $[10,11]$. Surgery is recommended for all main-duct IPMN because of the higher malignant potential. In contrast, surgery for side-branch IPMN is performed only for lesions that are symptomatic with features suggestive of advanced stage or malignancy such as dilated main PD, mural nodules, or positive cytology. Thus, international consensus guidelines for the treatment of branch duct IPMN were established to assist in decision making of pursuing the surgical option of resection (Table 1) $[12,13]$ although controversy remains over the optimal management [14].

Although pancreatobiliary and pancreatointestinal fistulae associated with IPMN of the pancreas have been reported in the elderly population, no such cases have been reported in middle aged patients and there are no reports making the connection between formation of the fistulae and subsequent reduction in the frequency of recurrent acute pancreatitis. The average age of patients with fistula formation is the late seventh decade and early eighth decade with a predominance of female gender [15]. Fistula formation is most common in the duodenum, 
Table 1. Summary of the International Consensus Guidelines and Multi-Institutional Study for the Treatment of Main Duct and Branch Duct IPMN

\begin{tabular}{llll}
\hline Management & 2006 Sendai Consensus Guidelines & 2012 Revision of Guidelines & 2017 Multi-institutional study \\
Main duct & $\begin{array}{l}\text { Resect the main duct and mixed } \\
\text { variant IPMNs as long as the patient } \\
\text { is a good surgical candidate with } \\
\text { a reasonable life expectancy. }\end{array}$ & $\begin{array}{l}\text { Rest all surgically fit patients } \\
\text { with main duct IPMN. }\end{array}$ & $\begin{array}{l}\text { The presence of obstructive jaundice, } \\
\text { lymphadenopathy, or pancreatitis had the } \\
\text { greatest strength in predicting the presence } \\
\text { of high grade dysplasia and invasive cancer. }\end{array}$ \\
Branch duct & $\begin{array}{l}\text { Asymptomatic: observation } \\
\text { "Worrisome features"a },\end{array}$ & $\begin{array}{l}\text { Having multiple worrisome features was } \\
\text { additive in predicting high grade dysplasia } \\
\text { and invasive cancer, with each additional } \\
\text { feature increasing the OR by 1.39. }\end{array}$ \\
& $\begin{array}{l}\text { Symptomatic: resection not only to } \\
\text { alleviate the symptoms, but also because } \\
\text { are introduced. } \\
\text { of a higher likelihood of malignancy. }\end{array}$ & $\begin{array}{l}\text { More conservative approach: } \\
\text { Branch duct IPMN of }>\end{array}$ & $\begin{array}{l}3 \text { cm without "high-risk } \\
\text { stigmata" can be observed } \\
\text { without immediate resection. }\end{array}$ \\
& \\
& \\
$\begin{array}{l}\text { Individualize decision to treat based } \\
\text { on patient preferences and willingness } \\
\text { to undergo follow-up studies, and the } \\
\text { availability of safe pancreatic resection. }\end{array}$ &
\end{tabular}

aPancreatitis, cyst size $>3 \mathrm{~cm}$, thickened or enhancing cyst wall, non-enhancing mural nodule, duct caliber change, pancreas atrophy, and main duct size between 5 - $9 \mathrm{~mm}$ is considered a "worrisome feature". bMPD diameter of $\geq 10 \mathrm{~mm}$ is one of the "high-risk stigmata".

followed by the stomach, common bile duct, and colon [16]. Furthermore, malignant IPMNs are more likely to develop fistulae. Patients with pancreatobiliary fistulae may present with obstructive jaundice and cholangitis which may be a result of one or combination of three potential mechanisms: direct malignant invasion of the IPMN, or mechanical compression by the cystic lesion in the head of the pancreas, or mucin impaction within the lumen of the bile duct $[14,15]$. Similarly, pancreatointestinal fistulae often result from mechanical pressure of the cystic lesion leading to erosion into the gastrointestinal lumen, or direct malignant invasion $[14,15]$.

Our case highlights two unique points. First, there exists limited literature describing the formation of the pancreaticoduodenal fistula from the pancreatic neck to the first portion of the duodenum in the setting of IPMN. This fistula decreased the frequency of recurrent acute pancreatitis attacks secondary to providing a better drainage of the mucus and debris from the main PD. The patient was doing well at follow-up and denied any further attacks. Taking into consideration the location of the pancreatitis, affecting the groove, the fistula likely occurred in the setting of chronic peri-pancreatic inflammation. Review of the last MRCP further supports fistula formation, as it demonstrates one of the PD side branches abutting the gastric body with significant gastric wall thickening. Second, pancreaticoduodenal fistula formation complicates surgical planning. In this scenario, presence of the fistula increased the morbidity associated with the surgical treatment option due to significant adhesions between the pancreas and the surrounding organs. Future studies are needed to better characterize the optimal management of such cases.

Physicians need to maintain a high index of suspicion for complications of IPMN such as fistula formation between the pancreas and surrounding structures in the setting of chronic inflammatory changes due to recurrent episodes of pancreatitis. Early identification of a fistula is important to prevent premature referral for surgical resection since such cases may not be amenable to surgical resection. Furthermore, recent studies have also suggested a higher incidence of additional primary malignancies in patients with IPMN of the pancreas compared to the general population [17]. Commonly reported sites include colorectal, breast, prostate, urinary tract, and lung in order of decreasing incidence. Although this may not have implications in elderly patients, in which IPMN are common, patients who are middle-aged may require consideration for close screening for other primary malignancies.

\section{Acknowledgments}

Part of this paper was presented in a poster format in the American College of Gastroenterology annual meeting on October 2018. The authors thank and acknowledge Dr. Danielle H. Carpenter, associate professor of Pathology, Saint Louis University School of Medicine, St. Louis, MO, USA, for providing and reviewing the pathology slide.

\section{Funding Disclosure}

No funding or financial support was received for this manuscript.

\section{Informed Consent}

Informed patient consent was obtained for publication of this 
case report.

\section{Author Contributions}

All authors contributed equality in drafting, writing and critical revision of the manuscript. All authors have reviewed the final version of the manuscript and approved submission.

\section{References}

1. Fong ZV, Fernandez-Del Castillo C. Intraductal Papillary Mucinous Neoplasm of the Pancreas. Surg Clin North Am. 2016;96(6):1431-1445.

2. Tanaka M, Kobayashi K, Mizumoto K, Yamaguchi K. Clinical aspects of intraductal papillary mucinous neoplasm of the pancreas. J Gastroenterol. 2005;40(7):669675.

3. Yamaguchi K, Tanaka M. Intraductal papillary-mucinous tumor of the pancreas: a historical review of the nomenclature and recent controversy. Pancreas. 2001;23(1):1219.

4. Basturk O, Hong SM, Wood LD, Adsay NV, AlboresSaavedra J, Biankin AV, Brosens LA, et al. A revised classification system and recommendations from the baltimore consensus meeting for neoplastic precursor lesions in the pancreas. Am J Surg Pathol. 2015;39(12):1730-1741.

5. Klibansky DA, Reid-Lombardo KM, Gordon SR, Gardner TB. The clinical relevance of the increasing incidence of intraductal papillary mucinous neoplasm. Clin Gastroenterol Hepatol. 2012;10(5):555-558.

6. Chang YR, Park JK, Jang JY, Kwon W, Yoon JH, Kim SW. Incidental pancreatic cystic neoplasms in an asymptomatic healthy population of 21,745 individuals: Largescale, single-center cohort study. Medicine (Baltimore). 2016;95(51):e5535.

7. Pergolini I, Sahora K, Ferrone CR, Morales-Oyarvide V, Wolpin BM, Mucci LA, Brugge WR, et al. Long-term risk of pancreatic malignancy in patients with branch duct intraductal papillary mucinous neoplasm in a referral center. Gastroenterology. 2017;153(5):1284-1294 e1281.

8. Morales-Oyarvide V, Fong ZV, Fernandez-Del Castillo C, Warshaw AL. Intraductal papillary mucinous neoplasms of the pancreas: strategic considerations. Visc Med. 2017;33(6):466-476.
9. Campbell NM, Katz SS, Escalon JG, Do RK. Imaging patterns of intraductal papillary mucinous neoplasms of the pancreas: an illustrated discussion of the International Consensus Guidelines for the Management of IPMN. Abdom Imaging. 2015;40(3):663-677.

10. Terris B, Ponsot P, Paye F, Hammel P, Sauvanet A, Molas $G$, Bernades $P$, et al. Intraductal papillary mucinous tumors of the pancreas confined to secondary ducts show less aggressive pathologic features as compared with those involving the main pancreatic duct. Am J Surg Pathol. 2000;24(10):1372-1377.

11. Bernard P, Scoazec JY, Joubert M, Kahn X, Le Borgne J, Berger F, Partensky C. Intraductal papillary-mucinous tumors of the pancreas: predictive criteria of malignancy according to pathological examination of 53 cases. Arch Surg. 2002;137(11):1274-1278.

12. Tanaka M, Chari S, Adsay V, Fernandez-del Castillo C, Falconi M, Shimizu M, Yamaguchi K, et al. International consensus guidelines for management of intraductal papillary mucinous neoplasms and mucinous cystic neoplasms of the pancreas. Pancreatology. 2006;6(1-2):1732.

13. Tanaka M, Fernandez-del Castillo C, Adsay V, Chari S, Falconi M, Jang JY, Kimura W, et al. International consensus guidelines 2012 for the management of IPMN and MCN of the pancreas. Pancreatology. 2012;12(3):183197.

14. Wilson GC, Maithel SK, Bentrem D, Abbott DE, Weber $\mathrm{S}$, Cho C, Martin RC, et al. Are the current guidelines for the surgical management of intraductal papillary mucinous neoplasms of the pancreas adequate? A multi-institutional study. J Am Coll Surg. 2017;224(4):461-469.

15. Ravaud S, Laurent V, Jausset F, Cannard L, Mandry D, Oliver A, Claudon M. CT and MR imaging features of fistulas from intraductal papillary mucinous neoplasms of the pancreas to adjacent organs: A retrospective study of 423 patients. Eur J Radiol. 2015;84(11):2080-2088.

16. Kobayashi G, Fujita N, Noda Y, Ito K, Horaguchi J, Obana T, Koshida S, et al. Intraductal papillary mucinous neoplasms of the pancreas showing fistula formation into other organs. J Gastroenterol. 2010;45(10):1080-1089.

17. Ishida M, Egawa S, Kawaguchi K, Aoki T, Sakata N, Mikami Y, Motoi F, et al. Synchronous and metachronous extrapancreatic malignant neoplasms in patients with intraductal papillary-mucinous neoplasm of the pancreas. Pancreatology. 2008;8(6):577-582. 